\title{
Correlation between Gingival Phenotype, Residual Ridge Height and the Schneiderian Membrane
}

\author{
BC Deepthi, Shilpa Shetty, CL Satish Babu, P Rohit, DM Mallikarjuna, R Bharat Raj
}

\begin{abstract}
Purpose: Sinus perforations are one of the most common complications during sinus augmentation procedures. Preoperative prediction of the antral membrane thickness would be of practical importance and serves as an additional information for surgical planning. The purpose of this study was to analyze a possible correlation and comparison between gingival phenotype and the residual ridge height on the dentulous and the edentulous side to the thickness of the healthy sinus mucosa.
\end{abstract}

Materials and methods: Twenty-five consecutive patients were included in the study; preoperative cone beam computed tomography $(\mathrm{CBCT})$ scanning was performed to radiographically evaluate the gingival phenotype (GP), the height of the residual ridge $(\mathrm{RRH})$ at the edentulous side and the dentulous side and thickness of the schneiderian membrane (SMT). The smallest $\mathrm{RRH}$, highest SM and gingival thickness values were recorded. These values were classified as follows: $\mathrm{RRH}<$ or $>3.5 \mathrm{~mm}$; $\mathrm{SM}<$ or $>1 \mathrm{~mm}$; and $\mathrm{GP}<1.5$ or $>2 \mathrm{~mm}$.

Results: On the edentulous side, Strong correlations of GP with SM $(r=0.833, p \leq 0.001)$ and moderate between RRH with SM $(r=0.620, p=0.001)$ and RRH with GP $(r=0.596$, $p=0.001)$ were noted. On the dentulous side, strong correlations of GP with SM $(r=0.832, p \leq 0.001)$ and moderate between RRH with SM $(r=0.643, p=0.001)$ and RRH with GP ( $r=$ $0.601, p=0.001$ ) were noted.

Conclusion: Within the limitations of the study, the gingival phenotype (GP) presents to be a reliable measure to predict the thickness of the SM and suggested that the GP, SM, RRH may be important factors for sinus perforation.

Keywords: Schneiderian membrane, Gingival phenotype, Residual ridge height, Sinus perforation, Computed tomography.

How to cite this article: Deepthi BC, Shetty S, Satish Babu $\mathrm{CL}$, Rohit P, Mallikarjuna DM, Bharat Raj R. Correlation between Gingival Phenotype, Residual Ridge Height and the Schneiderian Membrane. Int J Oral Implantol Clin Res 2012;3(3): 111-115.

\section{Source of support: Nil}

Conflict of interest: None declared

\section{INTRODUCTION}

Implant placement in the posterior maxilla is frequently problematic because of the extension of the maxillary sinus into the alveolar ridge area. ${ }^{1}$ The Schneiderian membrane (SM) lines the sinus internally with a thin mucosa of ciliated respiratory epithelium which is continuous with that of the nose; however, it is thinner and less vascular than the nasal mucosa. ${ }^{2}$ Maxillary sinus augmentation offers a predictable treatment modality to increase the bone volume available for posteriorly placed oral implants. During sinus lift surgeries, the elevation of the SM is a delicate procedure as the membrane constitutes as important barrier for the protection of the sinus cavity. To reduce the risk of infections and optimize clinical outcomes in term of bone regeneration, its integrity should be preserved as much as possible. ${ }^{3}$

The most common intraoperative complication of sinus lift procedure is perforation of the SM. ${ }^{4}$ At single implant sites it is particularly important to correlate the extension of the sinus lifting with the sinus membrane deformation capacity as well as the thickness. Clinical observations suggest a correlation between the sinus membrane thickness and the risk of perforations. ${ }^{5}$ And also between the thickness of the membrane and the residual alveolar ridge height and membrane perforation. Several anatomic factors have been implicated in the risk of sinus membrane perforation, particularly inadequate residual ridge height ( $\mathrm{RRH}$ ) or thinned sinus membrane. ${ }^{2,6}$ Sinus pathologies and certain medications may alter the membrane thickness (MT). ${ }^{2}$ However, data regarding the anatomic factors that affect the thickness of healthy sinus mucosa are limited. Recently, Aimetti et $\mathrm{al}^{7}$ reported that healthy sinus membranes are thicker in subjects with a thick gingival biotype than in those with a thin gingival biotype. Further, Ochsenbein and Ross ${ }^{8}$ indicated that there are two main types of gingival phenotype (GP), namely the scalloped and thin, the flat and thick gingiva. They reported that the gingival form closely follows that of the underlying alveolar bone. Although it has been suggested that the thickness of the marginal periodontium is genetically determined, no knowledge is yet available concerning the sinus mucosa. ${ }^{7}$ Pneumatization of the sinus may limit the amount of residual bone. Given the tendency of the alveolar ridge to progressively resorb after tooth loss and the typically poorer quality of posterior maxillary bone, rehabilitation of this area with implant supported fixed prostheses has traditionally been challenging. ${ }^{9}$ Hence, gingival phenotypes and the residual alveolar ridge height on the dentulous and edentulous side may assist the clinician in addressing the features of the Schneiderian mucosa. However, there are very less reports on the relationships among the gingival phenotype (GP), residual alveolar ridge (RRH), SM thickness and comparison between the dentulous and the edentulous sides. Therefore, the aim of 
this preliminary study was to determine the correlations among GP, RRH, MT on both edentulous and dentulous sides.

\section{MATERIALS AND METHODS}

Twenty-five consecutive systemically healthy patients with maxillary right missing molar (15 men and 10 women), with a mean age of $31.55 \pm 5.5$ years (range: $25-45$ ), were included in the study. During enrollment, the following inclusion criteria were considered: Clinically (1) healthy gingiva, (2) absence of bleeding on probing, (3) presence of all the teeth except 16, (4) no periodontal probing depth in excess of $3 \mathrm{~mm}$. The exclusion criteria were: ${ }^{10}$ (1) maxillary sinus pathologies/history of sinus surgery, (2) medication like phenytoin, (3) history of periodontal surgery, (4) smokers, (5) pregnancy and lactation. Preoperative computed tomographic scans and periapical radiographs were obtained to confirm the absence of maxillary sinus mucosal thickening and to evaluate dental as well as periodontal conditions.

All patients participating in this study were subjected to 3D cone beam computed tomography (CBCT), coronal and sagittal images with $1 \mathrm{~mm}$ slice thickness were obtained to measure the thickness of the SM, GP and RRH on both right and left sides. $\mathrm{RRH}$, was determined by drawing a reference line on the CT image from the lowest point of the sinus floor to the highest point of the alveolar ridge; the recorded values were classified as greater or lesser than 3.5 mm. ${ }^{11}$ The membrane thickness (SM), was measured at the center of the inter-teeth distance (proposed implant site). For each sinus, the highest values of SM was recorded and classified as greater than or lesser than $1 \mathrm{~mm}$ (Figs 1 and 2). ${ }^{11}$

The soft tissue thickness (GP) was measured at $2.0 \mathrm{~mm}$ apically from the alveolar bone crest and perpendicular to the external cortical plate of the tooth socket by using the cross-sectional images taken at the midline of selected teeth.

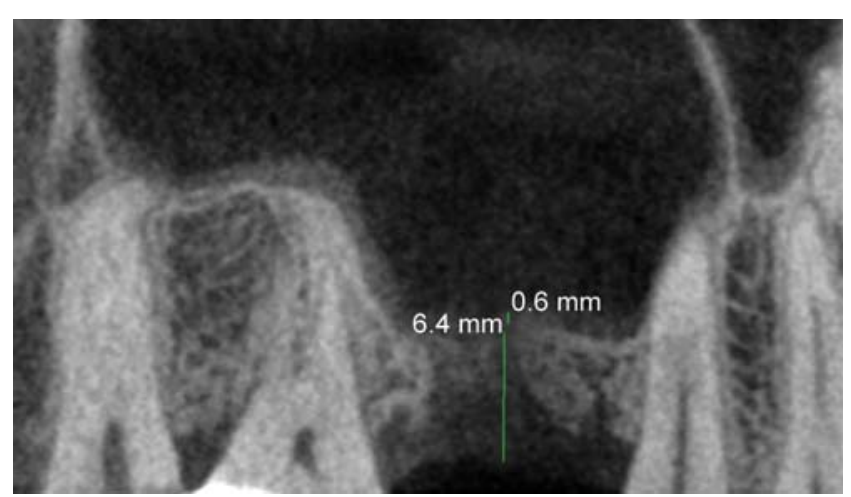

Fig. 1: Coronal CBCT slice showing the measurement of the residual ridge height and the sinus membrane thickness at the edentulous area

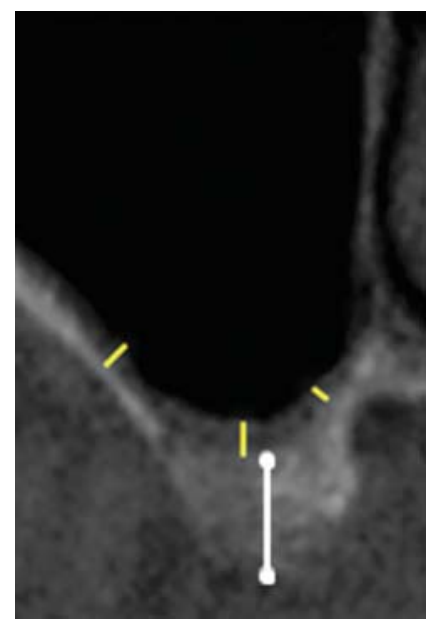

Fig. 2: Cross-sectional view of a CBCT image at the edentulous area. The white line shows $\mathrm{RRH}$, and the yellow lines show sinus membrane thickness

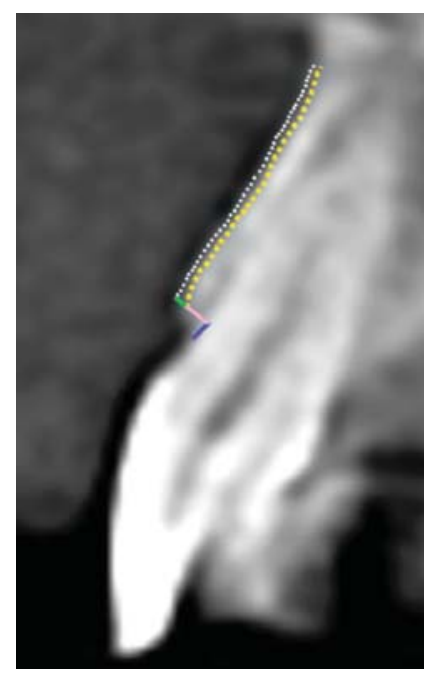

Fig. 3: Cross-sectional view of a СВCT image is used to evaluate gingival thickness. The yellow dashed lines indicate the border between soft and hard tissues. The blue line illustrates a distance of $2.0 \mathrm{~mm}$ from the alveolar bone crest, and the green line shows gingival thickness

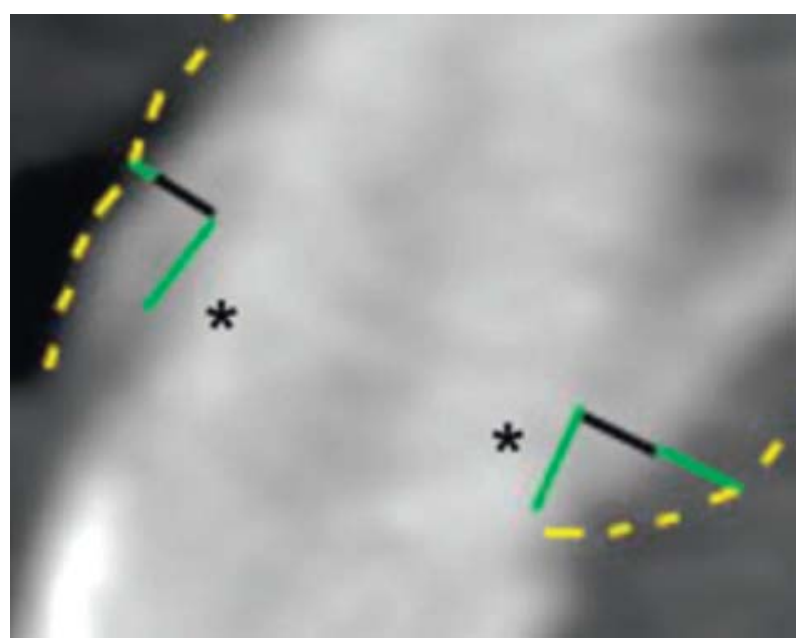

Fig. 4: Sagittal CBCT slice-gingival phenotype measured $2 \mathrm{~mm}$ apical to the alveolar bone crest (J Periodontol 2010;81:569-574) 


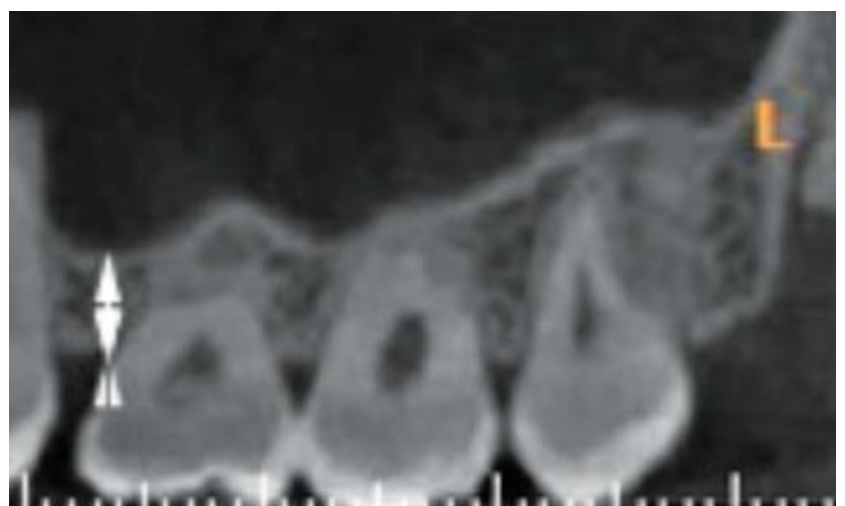

Fig. 5: Coronal CBCT slice showing the measurement of the residual ridge height of the dentulous side

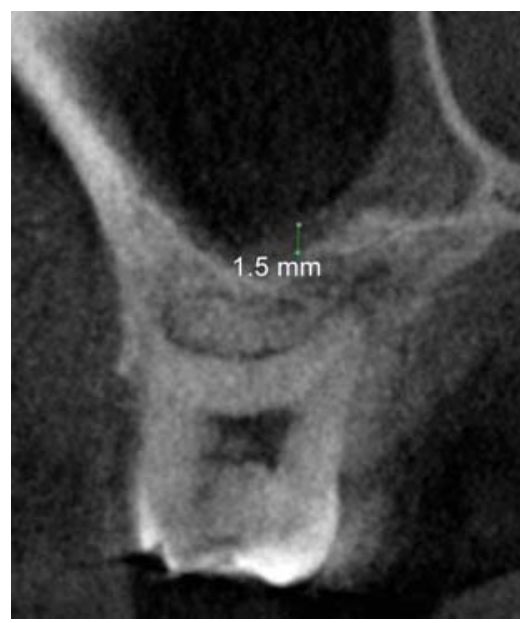

Fig. 6: Sagittal CBCT slice showing the measurement of the Schneiderian membrane of the dentulous side

GP was classified as either thin $(<1.5 \mathrm{~mm})$ or thick (>2 mm) (Figs 3 and 4). ${ }^{12,13}$

The measurements for RRH, SM and GP was repeated on the dentulous side the same way as the edentulous side (Figs 5 and 6). Then the measurements of the dentulous area were compared with the edentulous area.

\section{Statistical Analysis}

Chi-square test was used to determine the relationships between the parameters. Values of $\mathrm{p}<0.05$ were accepted as statistically significant. The strength of the correlation was determined by using the Pearson's r value: Mild correlation, $\mathrm{r}<0.40$; moderate correlation, $\mathrm{r}=0.40$ to 0.70 ; and strong correlation $r>0.70$.

\section{RESULTS}

The mean and standard deviations of the GP, RRH and SM values and the frequencies of these parameters are presented in Table 1. A strong correlation was found between GP and
SM $(r=0.832, p=0.001)$ on the dentulous side and edentulous side $(r=0.833, p=0.001)$.

The correlation between dentulous and edentulous side was found to be positive and very strong for RRH ( $r=0.898)$ and SM $(r=0.943)$ and the correlation was found to be statistically significant $(\mathrm{p}<0.001)$. It was also found to be positive and strong for GP $(r=0.781)$ and was found to be statistically significant ( $<0.001$; Table 1 and Graph 1 ).

The correlation between RRH and SM was found to be positive and moderate $(r=0.643)$, it was also statistically significant $(\mathrm{p}<0.01)$.

The correlation between RRH and SM was found to be positive and moderate $(r=0.601)$, it was also statistically significant $(\mathrm{p}<0.01)$.

The correlation between SM and GP was found to be positive and very strong $(r=0.832)$, it was also statistically significant ( $<<0.01$; Table 2 and Graph 2).

The correlation between RRH and SM was found to be positive and moderate $(r=0.620)$, it was also statistically significant $(\mathrm{p}<0.001)$.

The correlation between RRH and SM was found to be positive and moderate $(r=0.596)$, it was also statistically significant ( $<0.001$ ) (Table 3 and Graph 3).

The correlation between SM and GP was found to be positive and very strong $(r=0.833)$, it was also statistically significant $(\mathrm{p}<0.01)$.

Table 1: Correlation between dentulous and edentulous sides

\begin{tabular}{ccc}
\hline Parameter & $r$ & $p$-value \\
\hline RRH & 0.898 & $<0.001^{*}$ \\
SM & 0.943 & $<0.001^{*}$ \\
GP & 0.781 & $<0.001^{*}$ \\
\hline
\end{tabular}

*Denotes significant correlation

Table 2: Correlation between different parameters within dentulous side

\begin{tabular}{lll}
\hline Correlation between & $r$ & $p$-value \\
\hline $\mathrm{RRH}$ and SM & 0.643 & $0.001^{*}$ \\
$\mathrm{RRH}$ and GP & 0.601 & $0.001^{*}$ \\
$\mathrm{SM}$ and GP & 0.832 & $<0.001^{*}$ \\
\hline
\end{tabular}

*Denotes significant correlation

Table 3: Correlation between different parameters within edentulous side

\begin{tabular}{ccl}
\hline Correlation between & $r$ & $p$-value \\
\hline RRH and SM & 0.620 & $<0.001^{*}$ \\
RRH and GP & 0.596 & $<0.001^{*}$ \\
SM and GP & 0.833 & $<0.001^{*}$ \\
\hline
\end{tabular}

*Denotes significant correlation 


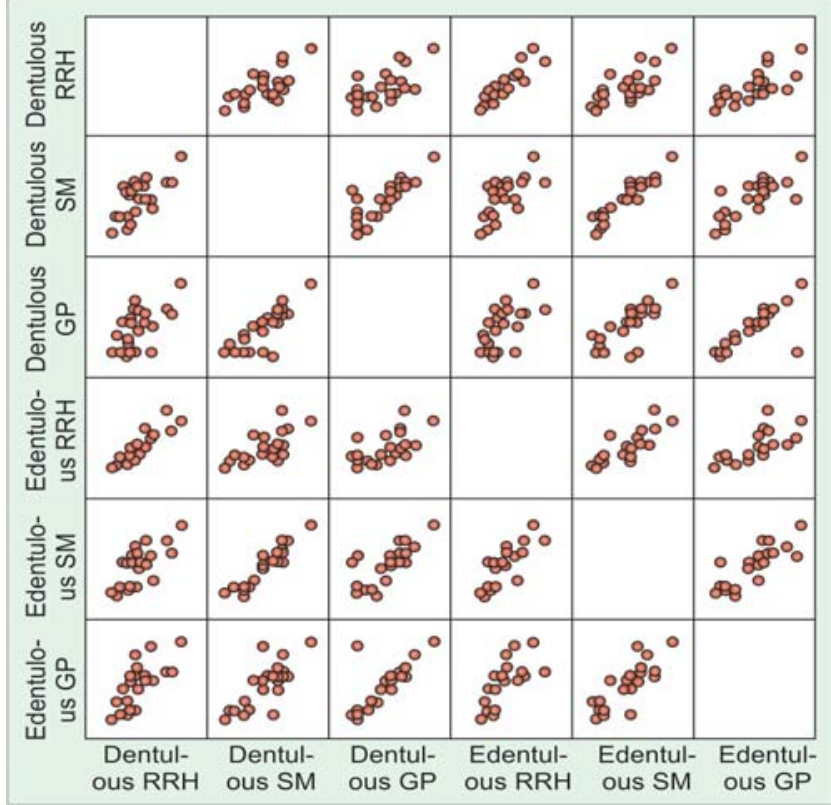

Graph 1: Correlation graph shows correlation between dentulous and edentulous sides for each parameter

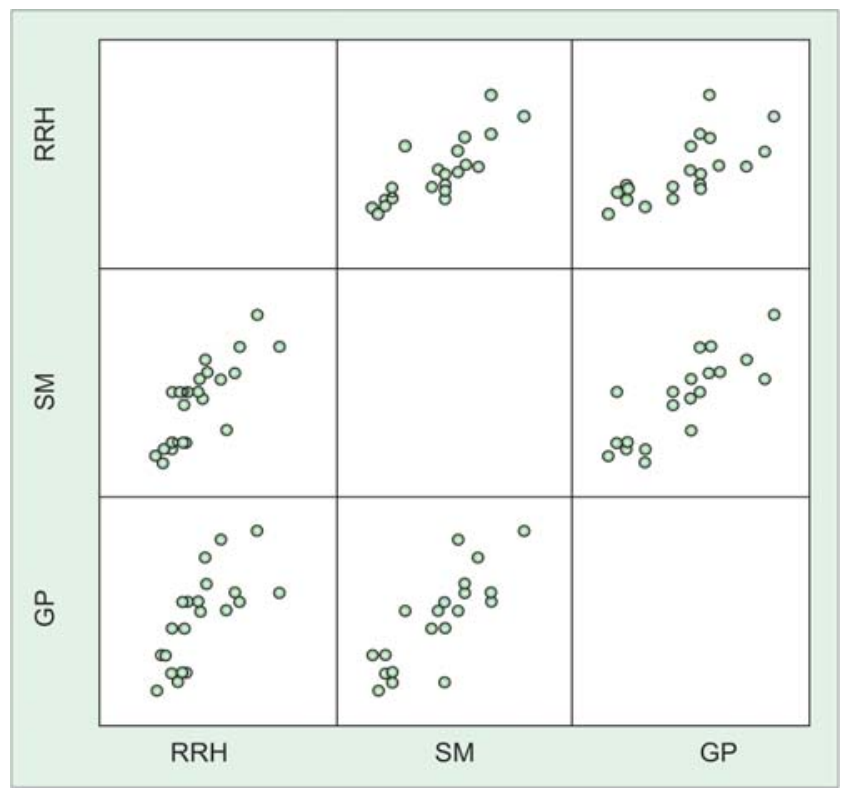

Graph 2: Correlation graph (edentulous group)

\section{DISCUSSION}

In developing a treatment plan for the maxilla that includes the posterior regions, the status of the maxillary sinus must be carefully considered. ${ }^{9}$ The maxillary sinus augmentation procedure has been well-documented, and implants placed in augmented bone have good long-term success/survival. ${ }^{14}$ Sinus membrane perforation is reported as the most common complication of this procedure. Membrane perforations, according to the literature, are strongly associated with the development of postoperative complications, mostly including of acute or chronic sinus inflammation, bacterial

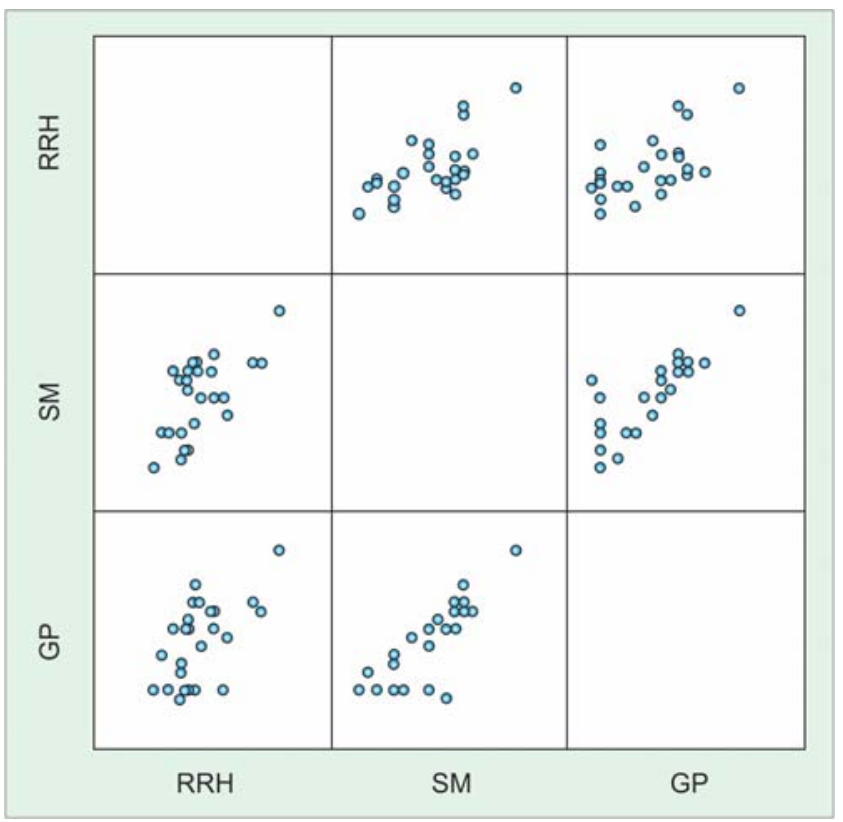

Graph 3: Correlation graph (edentulous group)

invasion, swelling, wound dehiscence and loss of graft material. ${ }^{15,16}$ Some studies show no difference in the success rate of implants placed with sinus bone grafting in patients whose SM was perforated versus those patients in whom the membrane remained intact. ${ }^{17}$ However, Shlomi et al ${ }^{18}$ suggested that repair of membrane with a resorbable collagen membrane may result in reduced bone formation and reduced implant survival. Pommer et al ${ }^{19}$ investigated the mechanical properties of the sinus membrane in vitro and showed that thicker membranes have significantly higher load limits. In the current study, SM strongly correlated with GP and moderately with RRH and showed a decrease in SM in the presence of RRH $<3.5 \mathrm{~mm}$ and a significant correlation between GP and SM $<1 \mathrm{~mm}$. A reason for these results is that the force necessary for membrane elevated from the bony sinus floor may not be endured by a thin sinus membrane.

Pommer, ${ }^{19}$ in his study also concluded that perforation of the SM occurred at mean tension of $7.3 \mathrm{~N} / \mathrm{mm}^{2}$. The membrane (mean thickness $0.9 \mathrm{~mm}$ ) could be stretched to $132.6 \%$ of its original size in one-dimensional elongation, and to $124.7 \%$ in two-dimensional elongation, which means a thick membrane can stretch to about one and a half times its original size, and this measurement can be correlated with thickness gingival phenotype clinically without any high end CBCT tool guide. And measuring the thickness of the gingiva by Muller's method, ${ }^{10}$ the thickness of SM can be assessed, which aids in minimizing the sinus membrane perforation during sinus lift as well as implant placement procedures. 
In the present study, CT imaging was used for sinus membrane and gingival thickness measurements, besides alveolar bone evaluation, because of the ability to magnify and change the brightness and contrast of the images using the computer program. Fu et $\mathrm{al}^{20}$ reported that CBCT measurements of both bone and labial soft tissue thicknesses are accurate and concluded that CBCT measurements might be a more objective method to determine the thickness of both soft and hard tissue than direct measurements.

\section{CONCLUSION}

The correlation between dentulous and edentulous region side was found to be positive. The thickness of the SM remained the same and correlated to the thickness of the GP on the dentulous and with missing tooth and reduced RRH.

Within the limitations of this study it may be suggested that thin GP is a risk factor for membrane perforation because of the correlation of GP with MT and RRH. This result is important clinically to predict risk factors for sinus membrane perforations during sinus-lift procedures, particularly if CT or CBCT evaluations are not possible.

\section{REFERENCES}

1. Del Fabbro M, Testori T, Francetti L, Weinstein R. Systematic review of survival rates for implants placed in the grafted maxillary sinus. Int J Periodontics Restorative Dent 2004 Dec;24(6):565-77.

2. van den Bergh JP, ten Bruggenkate CM, Disch FJ, Tuinzing DB. Anatomical aspects of sinus floor elevations. Clin Oral Implants Res 2000;11:256-65.

3. Proussaefs P, Lozada J, Kim J, Rohrer. Repair of the perforated sinus membrane with a resorbable collagen membrane: A human study. Int J Oral Maxillofac Implants 2004 May-Jun;19(3): 413-20.

4. Peleg M, Chaushu G, Mazor Z, Ardekian L, Bakoon M. Radiological findings of the post-sinus lift maxillary sinus: A computerized tomography follow-up. J Periodontol 1999;70:1564-73.

5. Berengo M, Sivolella S, Majzoub Z, Cordioli G. Endoscopic evaluation of the bone added osteotome sinus floor elevation procedure. Int J Oral Maxillofac Surg 2004 Mar;33(2):189-94.

6. Peleg M, Mazor Z, Garg AK. Augmentation grafting of the maxillary sinus and simultaneous implant placement in patients with 3 to $5 \mathrm{~mm}$ of residual alveolar bone height. Int J Oral Maxillofac Implants 1999;14:549-56.

7. Aimetti M, Massei G, Morra M, Cardesi E, Romano F. Correlation between gingival phenotype and Schneiderian membrane thickness. Int J Oral Maxillofac Implants 2008 NovDec;23(6):1128-32.

8. Ochsenbein C, Ross S. A reevaluation of osseous surgery. Dent Clin North Am 1969;13:87-102.

9. Testori T. Maxillary sinus surgery: Anatomy and advanced diagnostic imaging. Journal of Implant and Reconstructive Dentistry 2011;3(1).

10. Müller HP, Könönen E. Variance components of gingival thickness. J Periodontal Res 2005 Jun;40(3):239-44.
11. Yilmaz HG, Tözüm † TF. Are Gingival phenotype, residual ridge height and membrane thickness critical for the perforation of maxillary sinus? J Periodontol 2012 Apr;83(4):420-25.

12. Kan JY, Morimoto T, et al. Gingival biotype assessment in the esthetic zone: Visual versus direct measurement. Int J Periodontics Restorative Dent 2010 Jun;30(3):237-43.

13. Simone FM, Janner, et al. Characteristics and dimensions of the Schneiderian membrane: A radiographic analysis using cone beam computed tomography in patients referred for dental implant surgery in the posterior maxilla. Clinical Oral Implants Res 2011 Dec;22(12):1446-53.

14. Peleg M, Garg AK, Mazor Z. Predictability of simultaneous implant placement in the severely atrophic posterior maxilla: A 9-year longitudinal experience study of 2132 implants placed into 731 human sinus grafts. Int J Oral Maxillofac Implants 2006; 21:94-102.

15. Katranji A, Fotek P, Wang HL. Sinus augmentation complications: Etiology and treatment. Implant Dent 2008; 17:339-49.

16. Karabuda C, Arisan V, Hakan O. Effects of sinus membrane perforations on the success of dental implants placed in the augmented sinus. J Periodontol 2006;77:1991-97.

17. Ardekian L, Oved-Peleg E, Mactei EE, Peled M. The clinical significance of sinus membrane perforation during augmentation of the maxillary sinus. J Oral Maxillofac Surg 2006;64:277-82.

18. Shlomi B, Horowitz I, Kahn A, Dobriyan A, Chaushu G. The effect of sinus membrane perforation and repair with Lambone on the outcome of maxillary sinus floor augmentation: A radiographic assessment. Int J Oral Maxillofac Implants 2004;19:559-662.

19. Pommer B, Unger E, Sütö D, Hack N, Watzek G. Mechanical properties of the Schneiderian membrane in vitro. Clin Oral Implants Res 2009;20:633-37.

20. Fu JH, Yeh CY, Chan HL, Tatarakis N, Leong DJ, Wang HL. Tissue biotype and its relation to the underlying bone morphology. J Periodontol 2010;81:569-74.

\section{ABOUT THE AUTHORS}

\section{BC Deepthi (Corresponding Author)}

Postgraduate Student, Department of Prosthodontics, VS Dental College and Hospital, Bengaluru, Karnataka, India, e-mail: deepthibc@googlemail.com

\section{Shilpa Shetty}

Professor, Department of Prosthodontics, VS Dental College and Hospital, Bengaluru, Karnataka, India

\section{Satish Babu}

Professor and Head, Department of Prosthodontics, VS Dental College and Hospital, Bengaluru, Karnataka, India

\section{P Rohit}

Reader, Department of Prosthodontics, VS Dental College and Hospital, Bengaluru, Karnataka, India

\section{DM Mallikarjuna}

Postgraduate Student, Department of Prosthodontics, VS Dental College and Hospital, Bengaluru, Karnataka, India

\section{R Bharat Raj}

Senior Lecturer, Department of Prosthodontics, VS Dental College and Hospital, Bengaluru, Karnataka, India 